\title{
Invasive pressure monitoring saves from tuberculous meningitis with fulminant generalized brain edema
}

\section{George Trendelenburg1 ${ }^{*}$, Daniel Jussen², Steffen Grimmer ${ }^{3}$,Wibke Jakob², Nicola E. Hiemann ${ }^{4}$ and Peter Horn²}

1 Department of Neurology, Universitätsmedizin Göttingen, Göttingen, Germany

2 Department of Neurosurgery, Charité Universitätsmedizin Berlin, Berlin, Germany

${ }^{3}$ Anaesthesiology and Intensive Care Medicine, Charité Universitätsmedizin Berlin, Berlin, Germany

${ }^{4}$ Deutsches Herzzentrum Berlin, Berlin, Germany

Edited by:

S. Andrew Josephson, University of California, San Francisco, USA

\section{Reviewed by:}

Joshua Klein, Brigham and Women's Hospital, USA

Joseph D. Burns, Boston University, USA

\section{${ }^{*}$ Correspondence:}

George Trendelenburg, Deparment of Neurology, Universitätsmedizin Göttingen, Göttingen, Germany. e-mail: george.trendelenburg@med. uni-goettingen.de
We report a 57-year old female patient with a rapid and dramatic dynamic of whole brain edema caused by tuberculous meningitis. After initiation of tuberculostatic medication, general condition of the patient worsened and finally she was intubated due to a progredient loss of consciousness and respiratory insufficiency. Repeated cerebral computer tomography (CCT) revealed a global brain edema with slit ventricles and a dramatic progress of generalized brain swelling. Highly interesting, a rapid expanded regime of brain pressure monitoring and treatment according to a neurosurgical intensive standard ICP/CPP management protocol, which was complemented by the tuberculostatic therapy and high dose steroid application, dramatically improved the general conditions, so that the patient is now in a general condition which corresponds that before the occurrence of tuberculous meningitis. Thus, it is mandatory in situations with a rapid progressive brain swelling caused by bacterial meningitis to consider an intensified cerebral monitoring and stratified treatment protocol in order to avoid the devasting effects of a long lasting increase in intracranical pressure.

Keywords: meningitis, intracranial pressure, monitoring, cerebral perfusion pressure

\section{INTRODUCTION}

We examined a 57 -year-old female patient, who was receiving combined immunosuppressive medication, including tacrolimus, following a lung transplant 2 years previously.

The patient was initially hospitalized in the cardio-surgical department due to a generally decreasing state of health (diarrhea, weight loss). During the subsequent 2 weeks, this right-handed patient developed mild aphasic symptoms (difficulties of speech comprehension and amnesic aphasia), recurrent holocephalic headaches, visual disturbances (fluctuating visual field defects with photo- and phono-phobia), and a left hemiparesis. An analysis of the cerebro-spinal fluid revealed a mild pleocytosis (266 cells/ $\mu \mathrm{l})$ with a positive PCR test for M. tuberculosis. At the same time further microbiological and virological testing remained negative, including tests for the herpes virus.

Oral tuberculostatic medication was initiated, and the medication with tacrolimus and corticosteroids $(7 \mathrm{mg}$ p.d.) was continued. During the following days the general condition of the patient worsened, and finally she was intubated due to a progressive loss of consciousness and respiratory insufficiency. A cerebral computer tomography revealed a progressive global brain edema with compressed ventricles (Figure 1). Fourteen hours later a second cerebral computer tomography revealed a dramatically progressive generalized brain swelling; at this point the patient was transferred to the neurosurgical-neurological intensive care unit.

A neurological exam revealed a non-responsive tetraparetic patient with slow pupillary reflexes, though this last could also be attributed to the ongoing effects of the analgo-sedation.
Nevertheless, the velocity of the neurological decline during the previous days, in combination with the results of the cerebral computer tomography (Figure 1D-F), prompted us to initiate invasive intracranial pressure (ICP) monitoring through the use of an intraparenchymal microprobe (Neurovent-P, Raumedic, Germany) immediately on the patients arrival at the neuro-intensive care unit.

Initially, the ICP was critically elevated $(39.9 \mathrm{mmHg})$. A protocol driven ICP/cerebral perfusion pressure (CPP) management (ICP $<20 \mathrm{mmHg}, \mathrm{CPP}>65 \mathrm{mmHg}$ ) was initiated. In addition to carrying out routine measures (positioning, sedation, normotension, and analgesia) and elevated ICP was managed with osmotic therapy (100 ml NaCl 10\% and/or $125 \mathrm{ml}$ mannitol 20\% i.v.) in combination with mild hyperventilation $\left(\mathrm{pCO}_{2} 32-35 \mathrm{mmHg}\right.$ ) and increased doses of sedation. To control inflammatory-induced brain swellings, high dose glucocorticoids ( $60 \mathrm{mg}$ dexamethasone i.v., followed by three times $8 \mathrm{mg}$ p.d., and a single additional dose of $40 \mathrm{mg}$ in critical episodes) were applied in combination with hypertonic saline infusions (target sodium $145-155 \mathrm{mmol} / \mathrm{l}$, target osmolality $310-320 \mathrm{mOsm} / \mathrm{kg}$ ) whenever the ICP exceeded a $20 \mathrm{mmHg}$ mark. The doses of the sedating therapy were intensified depending on the requirements of the elevated brain pressure (the midazolam dose was increased from $0.15 \mathrm{mg} / \mathrm{kg} / \mathrm{h}$ to $0.3 \mathrm{mg} / \mathrm{h}$ and additional $500 \mathrm{mg}$ thiopental injections were applied), and a combined tuberculostatic therapy with rifampicin (i.v.), streptomycin (i.v.), isoniazid (i.v.), ethambutol (i.v.), and pyrazinamid (p.o.) was administered.

Eleven days after having been transferred to the intensive care unit, the computer tomography of the patient revealed only a marginal brain edema. Since the ICP remained normal while reducing 


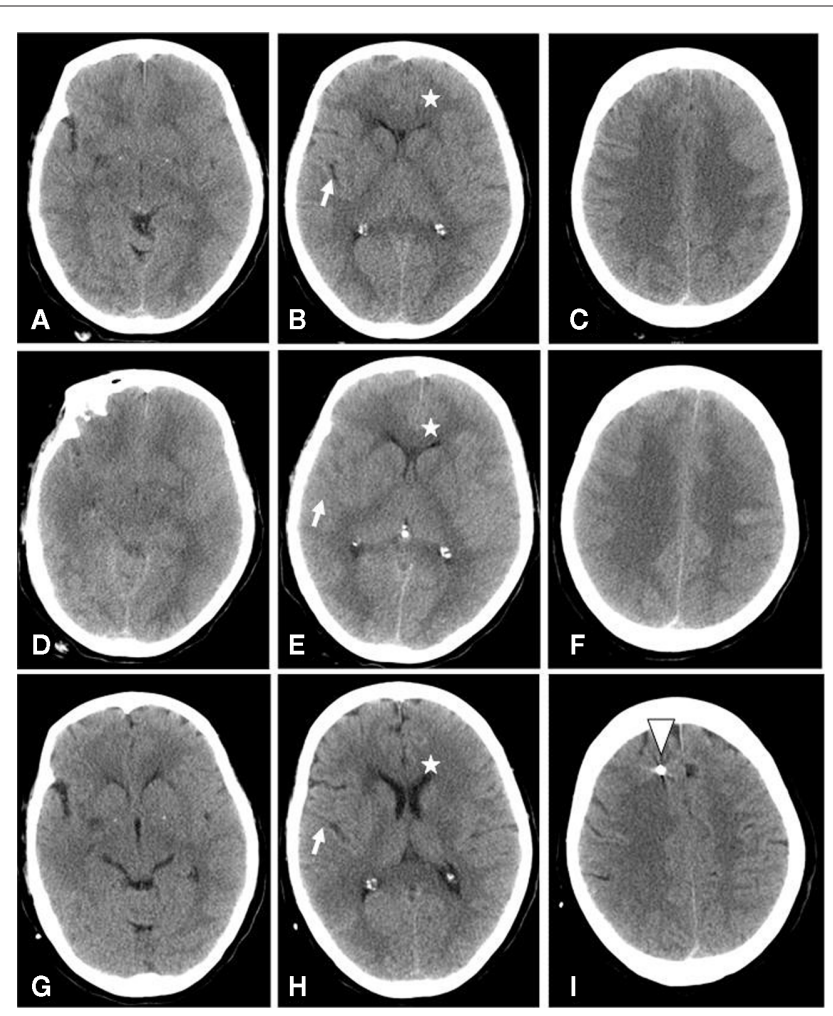

FIGURE 1 | Native cranial computer tomography at three different positions, performed before admission at $\mathbf{3}$ a.m. on day 1 (A-C), on the same day $14 \mathrm{~h}$ later (at 5 p.m.) (D-F), and 10 days after admission (G-I). Please note that brain sulci (white arrows) and ventricular spaces (white star) could be easily identified after treatment $\mathbf{( H )}$, but hardly during progress of brain swelling (B,E). White triangle in (I): position of the parenchymal pressure measurement catheter.

the sedative medication, the pressure sensor device was removed, and 2 weeks after her transfer the patient exhibited sufficient spontaneous respiratory function to allow her to be extubated. After 2 months, the patient was able to take part in her normal life, to discuss daily matters with relatives and to arrange everyday affairs on her own.

\section{BACKGROUND}

Tuberculous meningitis is the most lethal form of tuberculosis, mainly occurring in industrialized countries in immunocompromised individuals such as human immunodeficiency virus-infected patients or patients treated with immunosuppressive agents. The pathological process is characterized by meningeal inflammation, basal exudates, vasculitis, and hydrocephalus (Figaji and Fieggen, 2010; Garg, 2010). Even though a range of reports about treatment with corticosteroids exists, there is a surprising lack of information with regard to neurosurgical therapy options during the early progression of the disease, which is often complicated by rapid generalized brain edema (Thwaites and Tran, 2005; Figaji and Fieggen, 2010). As found with bacterial non-tuberculous meningitis, cerebral blood flow auto-regulation is probably also impaired in tuberculous meningitis, though the mechanism remains unclear (Murthy, 2005). Unfortunately, the mortality and morbidity of tuberculous meningitis are still exceptionally high (Garg, 2010).

\section{DISCUSSION}

This case illustrates the rapid and dramatic dynamic of whole brain edema caused by tuberculous meningitis. It is of significant interest that a rapidly expanded regime of brain pressure monitoring and a treatment according to a neurosurgical intensive standard ICP/CPP management protocol, complemented with the tuberculostatic therapy of high doses of steroids, was able to dramatically improve the general condition of the patient; this procedure was able to cure a critically life-threatening situation, despite the generalized brain edema and initial highly elevated ICP. As a result, the patient's general condition now corresponds to that experienced before the occurrence of tuberculous meningitis.

Whilst a score of less than eight on the Glasgow Coma Scale suggests a serious pathology and a possibly elevated ICP, there are no established guidelines on when to institute ICP monitoring for patients with tuberculous meningitis (Murthy, 2005). However, there are anecdotal reports about ICP monitoring and neurosurgical treatment of elevated ICP for patients with non-tuberculous meningitis, including decompressive hemicraniectomy and ventriculostomy (Baussart et al., 2006; Sala et al., 2009). In a recent retrospective study, the indication for ICP monitoring was mainly based on the level of consciousness (Edberg et al., 2011). Furthermore, the use of steroids is not only known to reduce the cerebral edema, but also the production of cytokines and other chemicals involved in the immunopathogenesis of tuberculous meningitis (Murthy, 2005). Results of the Cochrane database analysis revealed that an adjunctive steroid therapy is associated with fewer deaths and a reduced incidence of severe residual disability in tuberculous meningitis (Prasad et al., 2000). It is important to note however that steroid therapy was observed to have no effect on elevated ICP during a recent study of young children with tuberculous meningitis (Schoeman et al., 1997).

This case has demonstrated that despite the initial highly elevated brain pressure values and a generalized brain edema (Figure 1A-F), an intensified therapy, including invasive brain pressure monitoring, can be used to cure such a life-threatening disease. We are convinced that in this case the invasive brain pressure monitoring with combined therapy of the brain pressure, through the use of deep sedation, steroids, and osmotic substances, were the most essential agents of the rapid improvement observed in the patients' incidence of tuberculous meningitis.

\section{CONCLUDING REMARKS}

Our report illustrates that in situations of rapidly progressive brain swellings caused by bacterial meningitis, intensified cerebral monitoring, and a stratified treatment protocol should be considered, in order to avoid the devastating effects of a long lasting increase of ICP. 


\section{REFERENCES}

Baussart, B., Cheisson, G., Compain, M., Leblanc, P. E., Tadie, M., Benhamou, D., and Duranteau, J. (2006). Multimodal cerebral monitoring and decompressive surgery for the treatment of severe bacterial meningitis with increased intracranial pressure. Acta Anaesthesiol. Scand. 50, 762-765.

Edberg, M., Furenbring, M., Sjölin, J., and Enblad, P. (2011). Neurointensive care of patients with servere communityacquired meningitis. Acta Anaesthesiol. Scand. 55, 732-739.

Figaji,A.A., and Fieggen, A. G. (2010). The neurosurgical and acute care management of tuberculous meningitis: evidence and current practice. Tuberculosis 90, 393-400.
Garg, R. K. (2010). Tuberculous meningitis. Acta Neurol. Scand. 122, 75-90.

Murthy, J. M. K. (2005). Managment of intracranial pressure in tuberculous meningitis. Neurocrit. Care 2, 306-312.

Prasad, K., Volmink, J., and Menon, G. R. (2000). Steroids for treating tuberculous meningitis. Cochrane Database Sys. Rev. 2000, CD002244.

Sala, F., Abbruzzese, C., Galli, D., Grimaldi, M., Abate, M. G., Sganzerla, E. P., and Citerio, G. (2009). Intracranial pressure monitoring in pediatric bacterial meningitis: a fancy or useful tool ? A case report. Minerva Anestesiol. 75, 746-749.

Schoeman, J. F., Van Zyl, L. E., Laubscher, J. A., and Donald, P. R. (1997). Effect of corticosteroids on intracranial pres- sure, computed tomographic findings, and clinical outcome in young children with tuberculous meningitis. Pediatrics 99, 226-231.

Thwaites, G. E., and Tran, T. H. (2005) Tuberculous meningitis: many questions, too few answers. Lancet Neurol. 4, 160-170.

Conflict of Interest Statement: The authors declare that the research was conducted in the absence of any commercial or financial relationships that could be construed as a potential conflict of interest.

Received: 24 August 2011; accepted: 21 October 2011; published online: 15 November 2011.
Citation: Trendelenburg G, Jussen D, Grimmer S, Jakob W, Hiemann NE and Horn P (2011) Invasive pressure monitoring saves from tuberculous meningitis with fulminant generalized brain edema. Front. Neur. 2:69. doi: 10.3389/ fneur.2011.00069

This article was submitted to Frontiers in Neurocritical and Neurohospitalist Care, a specialty of Frontiers in Neurology.

Copyright (c) 2011 Trendelenburg, Jussen, Grimmer, Jakob, Hiemann and Horn. This is an open-access article subject to a nonexclusive license between the authors and Frontiers Media SA, which permits use, distribution and reproduction in other forums, provided the original authors and source are credited and other Frontiers conditions are complied with. 